\title{
The specific value of freedom
}

\author{
Martin van Hees
}

Received: 19 November 2008 / Accepted: 12 May 2010 / Published online: 29 May 2010

C The Author(s) 2010. This article is published with open access at Springerlink.com

\begin{abstract}
This article examines the question of how to assess our overall freedom in terms of its so-called specific value, i.e., the part of its value that can be reduced to the value of the various specific things we are free to do or be. It is argued that existing preference-based freedom rankings may fail to capture this value adequately and, drawing on earlier work by Puppe (J Econ Theory 68:174-199, 1996; in: Laslier et al. (eds.) Freedom in economics. New perspectives in normative analysis, 1998) and Puppe and $\mathrm{Xu}$ (Soc Choice Welf, this issue, 2010), an alternative, more general framework is proposed. Two rankings of freedom's specific value are axiomatically characterized. The article concludes with a discussion of possible extensions of the framework.
\end{abstract}

\section{Introduction}

Whether we talk about a person's well-being, try to make quality of life assessments or evaluate different institutional arrangements in terms of their impact on persons' lives, our judgements often not only reflect the value of the things a person is actually doing but will also depend on the value of the things that he or she could have been doing if she had so chosen. That is, being able to make a choice from among a set of options-having freedom of choice-has a value that is at least partly independent of the actual choice we make or intend to make from that set. For instance, it makes an important difference whether parents can choose from a number of options when selecting a school for their children. Similarly, when making a judgment about a person's well-being it makes a difference whether that person's poor health originates

M. van Hees $(\varangle)$

Faculty of Philosophy, University of Groningen, Oude Boteringestraat 52, 9712 GL Groningen,

The Netherlands

e-mail: Martin.van.Hees@rug.nl 
from a lack of economic means to eat healthily and to exercise regularly, or whether it was the result of a freely chosen hedonistic but unhealthy lifestyle.

To ascertain how well a person is doing we therefore cannot focus only on what she is doing; we should also consider what she could be doing. The range of one's choice options, i.e., the nature of one's opportunity set and thus of one's freedom of choice, is itself an ingredient of our well-being. ${ }^{1}$ For this reason, we would like to be able to make judgements about the value of our freedom: how much value do we derive from our opportunity set? Is it more valuable to be free to choose from this particular opportunity set rather than from another one? If so, why ${ }^{2}$ Following Ian Carter, we can make a distinction between two distinct types of reasons for valuing an opportunity set. ${ }^{3}$ The first type refers to the value of one's opportunity set insofar as that value is independent of the value of the elements of the set. That is, the opportunity set is taken to have at least some value which cannot be reduced to the value of the elements of the set. An example of such value, which is called the non-specific value of freedom, is the value that we may attach to responsibility. If it is of value to be responsible for one's choices, and if a person is only responsible for his choices if he could have chosen differently, then being able to make choices is in itself valuable. To illustrate, consider the value of a person's opportunity set which consists of only two alternatives, $x$ and $y$. Suppose, as observers, we do not have any information about the value of $x$ or $y$; we only know that the options represent two opposite courses of action and that the person can be held responsible for his eventual choice because he could have opted for the other alternative. If this responsibility is taken to be valuable, then being able to choose between $x$ and $y$ has value even though we know nothing about the value of $x$ and $y$ themselves. ${ }^{4}$

This article ignores such non-specific value and focuses on freedom's specific value only; that is, we want to compare opportunity sets in terms of their value insofar as that value can be reduced to the value of the elements of the sets.

\subsection{Freedom's specific value and existing freedom rankings}

It could be argued that various existing freedom rankings, in particular those that make use of preference information, already capture freedom's specific value. Whereas these approaches do indeed capture an important part of freedom's specific value, they may not be able to do so completely. ${ }^{5}$ Consider first the rather naive idea that an opportunity

\footnotetext{
1 Of course, I here follow the argumentation put forward by Amartya Sen; see e.g., Sen $(1985,1987,2004)$.

2 For a general overview of the ranking of opportunity sets, see Barberà et al. (2004). For an overview of the literature on freedom rankings, see Dowding and van Hees (2009).

3 Carter (1999). My rendition of the distinction differs, however, from that of Carter and is taken from Van Hees (2000).

4 Frankfurt (1969) famously criticized the idea that a person can only be held responsible for his actions if he could have acted differently. The point here, however, is not to defend a particular view of responsibility, but merely to give an illustration of freedom's non-specific value if one adheres to a particular theory of responsibility.

5 In the various preference-based approaches discussed below-indirect utility, effective freedom, expected indirect utility, reasonable persons' preferences - the value of an opportunity set is reducible to the value of
} 
set's specific value equals its indirect utility —a set $A$ is taken to have at least as much specific value as a set $B$ if, and only if, there is an alternative in $A$ which is considered to be at least as good as any element of $B$. The ranking is naive for two reasons. First, it fails to take account of the fact that we often attach value to our freedom precisely because we do not know our preferences. If I am not yet sure about which restaurant I want to visit tonight, I would rather keep my options open and not make a reservation yet. The idea that we value freedom because we have such a 'preference for flexibility' (Kreps 1979) can be captured in different ways. The effective freedom ranking presented by Foster (1992) looks at the various preferences a person could have (his 'potential preferences') and then deems a set $A$ to have at least as much value as a set $B$ if, and only if, for each of the person's potential preferences the set $A$ has at least as much indirect utility as the set $B$. Arrow (1995) captures the same intuition but adds information about the probability of the various potential preferences. He then ranks the various opportunity sets in terms of their expected indirect utility.

Whereas the value freedom has because we do not know our future preferences for sure can thus be taken account of by the more sophisticated indirect utility approaches of Foster and Arrow, there is a second drawback from which those more sophisticated versions also suffer. A person's actual or potential preferences need not track all of the reasons that we can have for valuing the various alternatives - one may attach value to some state of affairs even though one has no preference for it. Suppose for instance that you are not very fond of the works of Franz Kafka. Given the literature that you do like, you also know for sure that you will never develop a preference for it. Under any of the indirect utility approaches described above, we should say that adding the option of reading any of Kafka's stories or novels does not add to the specific value of your freedom. However, if we assume that reading Kafka is valuable even for someone who will never prefer to do so, then the possibility or impossibility of reading Kafka does affect the value of your freedom. The converse is also possible-a person may prefer something which he has no reason to value. Take the archetypical sadist who most prefers a state of the world $x$ in which he inflicts maximum pain on other persons. On both the indirect utility and the potential preference approaches we should say that the availability of $x$ adds to the value of the sadist's opportunity set.

Several proposals have been made to accommodate such divergence between one's actual and potential preferences on the one hand and the reasons for valuing something on the other. The important thing to note is that these proposals also make use of preference information, albeit not necessarily the preferences of the person whose freedom we are examining. Instead they are the preferences of the members of some reference class of individuals. For instance, Pattanaik and Xu (1998) use information about the preferences of reasonable persons. Sugden (1998) argues for using the

\section{Footnote 5 continued}

its elements in the sense that there is a functional relation between the relevant preference space (or, in case of Arrow's ranking, the space of preferences and their possible probabilities) and the freedom ranking. It is because of the existence of such a functional relation that I say they capture part of freedom's specific value rather than non-specific value. Note that this view on the nature of specific value differs from that of Carter (1999), who takes the value that we attach to freedom because of uncertainty about our future preferences to be an instance of freedom's non-specific value. 
preferences of individuals who share certain sociological characteristics with the person whose freedom we are assessing.

The shift from the indirect utility approaches to approaches that refer to the preferences of 'reasonable' or 'representative' persons goes a long way toward establishing freedom's specific value. Indeed, if we take the reasonable persons' preferences approach we see that the examples given above can be accommodated: gaining an extra option which the person in question would never consider choosing (reading Kafka) does enhance the value of a person's freedom, whereas the addition of an option that no reasonable person would ever consider choosing (the sadist's infliction of pain) will not do so. However, such agent-independent preferences may also fail to track the reasons we have for valuing an option if there is a distinction between there being a reason to value some option $x$ and there being a reasonable (representative) person who prefers $x$. Obviously, if such a distinction can indeed be sustained, then the use of the preferences of reasonable (or representative) persons will not capture all of the relevant reasons for valuing alternatives. ${ }^{6}$

\subsection{Eligibility}

We do not take a stance here with respect to the meta-ethical question of whether the reasons to value some option can always be tracked by the preferences of reasonable or representative persons. The issue is circumvented by using a formal framework that directly takes as input information about the things we have reasons to value; in this sense our approach is more general than a preference-based one. An additional reason for using such a more direct approach - though its analysis is not an aim of this article-is related to that other part of freedom's value: its non-specific value. As explained later, we may conjecture that an eligibility approach is able to capture both freedom's specific and non-specific value. A preference-based approach, however, will fail to do justice to freedom's non-specific value. ${ }^{7}$

We therefore take as given the existence of an eligibility function which assigns to each opportunity set a subset of eligible alternatives, that is, alternatives we have reason to value. The notion of eligibility was introduced in the freedom literature by Benn and Weinstein (1971), discussed by Day (1977) and later by Jones and Sugden (1982). In the freedom literature functions that can be interpreted as eligibility functions were introduced by Puppe $(1996,1998)$ and further explored in Puppe and Xu (2010), but the functions are formally choice functions; they assign to each set a nonempty subset. ${ }^{8}$ The framework here is slightly more general since it allows for the possibility of the assignment of the empty set.

\footnotetext{
6 See, for instance, Griffin (2008, pp. 111-113) for an argument for the view that such a distinction should indeed be made.

7 Carter (1996). See, however, Sen (1996) for a critical discussion of this last claim.

${ }^{8}$ Furthermore, an important difference between this article and Puppe (1996, 1998) and Puppe and Xu (2010) is that in the latter contributions a judgement about an alternative's eligibility —or essentiality as it is called there-is derived from a given freedom ranking of opportunity sets. Here the converse route is followed: we try to derive freedom rankings given judgements about the eligibility of alternatives.
} 
After having presented the formal framework in Sect. 2, Sect. 3 discusses various properties of eligibility functions. In Sects. 4 and 5 two 'eligibility rankings' are characterized, that is, rankings which are meant to capture the intuitive idea that the value of a set increases when a person has more reasons to value its elements. One ranking is a straightforward generalization of the much-discussed cardinality ranking introduced by Pattanaik and Xu (1990), the other ranking was introduced by Puppe (1996) and characterized earlier by Puppe and Xu (2010). In the concluding section we discuss some limitations of these rankings and some possible extensions of the framework.

\section{Eligibility functions}

Let $X$ be the universal non-empty set of alternatives, assumed to be finite. $\mathcal{Z}$ and $\mathcal{Z}^{0}$ denote the set of all non-empty subsets of $X$ and the set of all subsets of $X$, respectively. Let $\succeq$ be a binary relation defined over $\mathcal{Z}$, with $\succ$ and $\sim$ denoting its asymmetric and symmetric part, respectively. We shall call $\succeq$ a freedom ranking. There are different ways in which such a ranking can be interpreted. As explained in the introduction, the emphasis here is on the analysis of the value of the opportunity set, in particular, its specific value. $A \succeq B$ is therefore to be interpreted here as 'the freedom of choice that $A$ offers has at least as much specific value as that of $B$ '.

An eligibility function is a mapping which specifies for each opportunity set which of its elements are eligible and which are not:

Definition 2.1 $E$ is a mapping from $\mathcal{Z}$ to $\mathcal{Z}^{0}$ such that for all $A \in \mathcal{Z}, E(A) \subseteq A$.

Note that an eligibility function can assign the empty set to a set of alternatives. In other words, a set may fail to contain eligible alternatives. Whether a set may indeed not have an eligible element in it will depend on the interpretation of the eligibility notion. Roughly, we can distinguish two lines of interpretation. In the first interpretation, saying that an element is eligible or not is making a judgement about the goodness of the state of affairs which results from choosing that element. I may, for instance, say that the option of smoking cigarettes is ineligible because smoking is bad for my health. Alternatively, I can take the eligibility of an option to say something about the admissibility of choosing the option. The option's eligibility or ineligibility then describes whether one is allowed to choose it. We may call the first view, the one focusing on the goodness or badness of the states of affairs resulting from one's choices, the axiological interpretation of eligibility. The second interpretation, which refers to the admissibility or inadmissibility of those choices, is a deontic one.

It is reasonable to assume that an eligibility function can assign an empty set on an axiological interpretation, whereas this is probably not the case on a deontic interpretation. To illustrate with a particularly stark example, suppose an individual is confronted with a choice between two bads-say she is forced to choose between a very unhealthy and dangerous job or starvation. If we take the eligibility to refer only to the goodness of an outcome, then neither of the two options will be eligible. On the other hand, if we think about eligibility in terms of the admissibility of one's choices, then we could argue that at least one of the person's choice options is eligible. If not, we would say that any choice an individual makes is inadmissible, violating 
the principle that a person may not be obligated to do the impossible ('impossibilium nulla obligatio est').

Before turning to a discussion of the properties of an eligibility function, we conclude this section with two useful definitions.

Definition 2.2 Given some $E, x \in X$ is unconditionally (or context-independently) eligible iff for all $A \in \mathcal{Z}, x \in A$ implies $x \in E(A), x \in X$ is unconditionally (or context-independently) ineligible iff for all $A \in \mathcal{Z}, x \notin E(A)$.

Definition 2.3 For all $x, y \in X$, we say that

1. $x$ is weakly superior to $y$ iff $x \in E(\{x, y\})$;

2. $x$ and $y$ are co-ineligible iff for all $A$ containing both $x$ and $y,\{x, y\} \cap E(A)=\emptyset$.

\section{Properties of eligibility}

An eligibility function 'almost' is a choice function; it differs only in that it can assign the empty set to a set. As is well known from the choice literature, there is a close relation between consistency properties and the rationalizability of choice functions. For our purposes, it is helpful to explore the relation between the consistency properties of an eligibility function and its rationalizability. In this section we shall provide that analysis, that is, we formulate two rationalizability results for eligibility functions, which form the direct counterparts of two well-known rationalizability results for choice functions. ${ }^{9}$

To do so, we must define the relevant notions of consistency and rationalizability for eligibility functions. First, consider the following consistency requirements for eligibility functions:

Condition 3.1 $(\alpha)$ For all $A \in \mathcal{Z}$ and all $B \in \mathcal{Z}, E(A \cup B) \cap A \subseteq E(A)$.

Condition $3.2(\beta)$ For all $A, B \in \mathcal{Z}$, if $x, y \in E(A)$, then $x \in E(A \cup B)$ iff $y \in$ $E(A \cup B)$.

Condition $3.3(\gamma)$ For all $A, B \in \mathcal{Z}$ and all $x \in X: x \in E(A) \cap E(B) \Rightarrow x \in$ $E(A \cup B)$.

Condition 3.4 ( $\xi$ ) For all $A, B \in \mathcal{Z}$, if $E(A) \neq \emptyset$, then $E(A \cup B) \neq \emptyset$.

The first three conditions are straightforward adaptations of the corresponding conditions for choice functions (Sen 1971). For instance, $\alpha$ is the extension of Sen's Property $\alpha$; it here expresses that an eligible element remains eligible if one or more other elements are removed from the opportunity set. Similarly, $\beta$ and $\gamma$ are the counterparts of Sen's Property $\beta$ and $\gamma$ for choice functions, respectively. Note that $\xi$, which demands that the expansion of any set having eligible elements has at least some eligible elements itself, is trivially fulfilled by choice functions.

Next we extend the idea of rationalizability to eligibility functions.

\footnotetext{
${ }_{9}$ In particular, Propositions 3.1 and 3.2 form the counterparts of Theorems T.9 and T.8 of Sen (1971), respectively.
} 
Definition 3.1 Given some $E$, the base relation $R$ is defined as the binary relation over $X$ satisfying: for any $x, y \in X, x R y$ iff $x$ is weakly superior to or co-ineligible with $y$.

Definition 3.2 Let $E$ be an eligibility function and $R$ the corresponding base relation. We say $E$ is normal iff for all $A$ :

1. $E(A) \neq \emptyset: E(A)=\{x \in A \mid x R y$ for all $y \in A\}$

2. $E(A)=\emptyset$ : all $x \in A$ are unconditionally ineligible.

Proposition 3.1 An eligibility function $E$ is normal iff $E$ satisfies $\alpha, \gamma$, and $\xi$.

Proof Let $R$ be the base relation.

(a) $\Rightarrow$ : Assume $E$ is normal. Take $A \cup B$ with non-empty $E(A \cup B) \cap A$. If $E(A)$ is empty, each element of $A$ is unconditionally ineligible, contradicting non-emptiness of $E(A \cup B) \cap A$. By definition, $E(A \cup B) \cap A=\{x \in A \mid x R y$ for all $y \in$ $A \cup B\}$. In particular, $x \in E(A \cup B) \cap A$ implies $x R y$ for all $y \in A$. Since $E(A)$ is not empty, $x R y$ for all $y \in A$ implies $x \in E(A)$. Hence, $\alpha$ is satisfied. To prove $\gamma$, let $x \in E(A) \cap E(B)$. We then have $x R y$ for all $y \in A$ and $x R y$ for all $y \in B$, and thus $x R y$ for all $y \in A \cup B$. Since $x$ is not unconditionally ineligible, $E(A \cup B)$ is non-empty. Hence, from normality it follows that $x \in E(A \cup B)$. Finally, if $E(A \cup B)=\emptyset$, normality implies that all elements in $A \cup B$ are unconditionally ineligible, implying emptiness of $E(A)$. Hence, $\xi$ is also satisfied.

(b) $\Leftarrow$ : If $E(A)=\emptyset, \xi$ directly implies that for all $x \in A, x \notin E(\{x\})$. Hence, by $\alpha$, each $x \in A$ is unconditionally ineligible. Take $A$ with non-empty $E(A)$. For all $x \in E(A)$ and all $y \in A, x \in E(\{x, y\})$ by $\alpha$. Hence, $x R y$ for all $y$ which shows $E(A) \subseteq\{x \in A \mid x R y$ for all $y \in A\}$. Now take $x \in A$ such that $x R y$ for all $y \in A$. We want to show that $x \in E(A)$. Take arbitrary $y \in E(A)$. By $\alpha, y \in E(\{x, y\})$. Hence, $x$ and $y$ cannot be co-ineligible, and $x R y$ entails $x \in E(\{x, y\})$. Note also that we have $E(\{x\})=\{x\}$ by $\alpha$. Now take arbitrary $y \in A-E(A)$. For all such $y, E(\{x, y\}) \neq \varnothing$ because of $E(\{x\})=\{x\}$ and $\xi$. Hence, $x$ and $y$ are not co-ineligible, and $x R y$ therefore entails $x \in E(\{x, y\})$. Since it was already established that $x \in E(\{x, y\})$ for all $y \in E(A)$, we have $x \in E(\{x, y\})$ for all $y \in A$. Consequently, by $\gamma, x \in E(A)$ and thus $\{x \in A \mid x R y$ for all $y \in A\} \subseteq E(A)$.

Proposition 3.2 An eligibility function $E$ is normal and $R$ is an ordering iff $E$ satisfies $\alpha, \beta$, and $\xi$.

Proof $\Rightarrow$ : Given Proposition 3.1 it only has to be shown that $\beta$ is satisfied. Let $A$ be some set in $\mathcal{Z}$ having at least two distinct eligible elements, say $x, y \in E(A)$. Assume $x \in E(A \cup B)$ for some $B \in \mathcal{Z}$. By normality $x R z$ for all $z \in A \cup B$. Normality also implies $y R x$. Transitivity therefore entails $y R z$ for all $z \in E(A \cup B)$, which means that $y \in E(A \cup B)$.

$\Leftarrow$ : Since $\alpha, \beta$ and $\xi$ imply $\gamma$, Proposition 3.1 establishes normality. Reflexivity and completeness follow directly from the definition of $R$. To show transitivity holds, assume $x R y$ and $y R z$. We need to examine four possible cases (where $P$ and $I$ denote the a-symmetric and symmetric parts of $R$, respectively): (a) $x P y$ and $y I z$ (b) $x I y$ and $y I z$, (c) $x P y$ and $y P z$, and (d) $x I y$ and $y P z$. 
(a) Let $x P y$ and $y I z$. We then have $E(\{x, y\})=\{x\}$. By $\xi E(\{x, y, z\}) \neq \emptyset$ and by $\alpha, y \notin E(\{x, y, z\})$. If $E(\{y, z\}) \neq \varnothing$, then $y I z$ implies $E(\{y, z\})=\{y, z\}$. Since $y \notin E(\{x, y, z\}), z \notin E(\{x, y, z\})$ by $\beta$ which in turn means that $E(\{x, y, z\})=$ $\{x\}$. If $E(\{y, z\})=\emptyset$, then both $y$ and $z$ are unconditionally ineligible and non-emptiness of $E(\{x, y, z\})$ also yields $E(\{x, y, z\})=\{x\}$. Hence, we have $E(\{x, y, z\})=\{x\}$. Condition $\alpha$ then yields $x \in E(\{x, z\})$ and $\beta z \notin E(\{x, z\})$. Thus $x P z$.

(b) Let $x I y$ and $y I z$. First, assume either $E(\{x, y\})$ or $E(\{y, z\})$ is non-empty. Without loss of generality, let $E(\{x, y\})$ be non-empty. By normality $E(\{x, y\})=$ $\{x, y\}$. By $\xi E(\{x, y, z\}) \neq \emptyset$. Since $y \in E(\{x, y\}), \alpha$ and $\xi$ imply $E(\{y, z\}) \neq \emptyset$. This means that $y$ and $z$ or not co-ineligible, and $y I z$ thus entails $E(\{y, z\})=$ $\{y, z\} . E(\{x, y, z\}) \neq \varnothing$ and $\beta$ now imply $E(\{x, y, z\})=\{x, y, z\}$ which by $\alpha$ entails $E(\{x, z\})=\{x, z\}$, which had to be shown. Next let $E(\{x, y\})=$ $E(\{y, z\})=\emptyset$. Conditions $\alpha$ and $\xi$ together imply $E(\{x, z\})=\emptyset$ and thus $x I z$.

(c) If $x P y$ and $y P z$, then $\{x\}=E(\{x, y\})$ and $\{y\}=E(\{y, z\}) . E(\{x, y, z\}) \neq \varnothing$ because of $\xi$, and by $\alpha y \notin E(\{x, y, z\})$ and $z \notin E(\{x, y, z\})$. Hence, $x \in$ $E(\{x, y, z\})$ and $x \in E(\{x, z\})$ by $\alpha$. Since $z \notin E(\{x, z\})$ because of $\beta$, we have $x P z$.

(d) If $x I y$ and $y P z, E(\{y, z\})=\{y\}$ and $E(\{x, y\})=\{x, y\}$ or $\emptyset$. If $E(\{x, y\})=\emptyset$, we must by $\alpha$ have $E(\{x, y, z\})=\emptyset$, contradicting $\xi$. Hence, $E(\{x, y\})=\{x, y\}$. By $\alpha, z \notin E(\{x, y, z\})$ and by $\beta, x \in E(\{x, y, z\})$. Hence, and again by $\alpha$ and $\beta, E(\{x, z\}=\{x\}$ and $x P z$.

\section{The simple eligibility ranking}

Perhaps the simplest way to compare opportunity sets is to say that the assessment of an opportunity set only depends on the number of eligible elements which that particular set contains. We call such rankings internal. Whether an alternative which is eligible in $A$ is also eligible in $A \cup B$, for instance, is not relevant when using an internal ranking for comparing $A$ and $B$. Clearly, different internal eligibility rankings are possible. That is, we can formulate different ways of basing the assessment of a set on the number of eligible elements within that set. We here characterize one internal ranking, viz. the one according to which the more eligible elements a set has, the more valuable it is. ${ }^{10}$

Definition 4.1 (Simple eligibility ranking) For all $A, B \in \mathcal{Z}, A \succeq \# B \Leftrightarrow \# E(A) \geq$ $\# E(B)$.

Clearly, if any element of any opportunity set is taken to be eligible, the ranking coincides with the simple cardinality ranking presented by Pattanaik and Xu (1990). That is, the more elements an opportunity set has, the higher it is ranked. Dissatisfied with the simple cardinality ranking, Pattanaik and Xu (1998) later presented a ranking

\footnotetext{
10 An alternative internal ranking, for instance, could be based on the ratio between the number of eligible elements and the total number of elements in the set. Cf. Steiner (1994) for a defence and Van Hees (1998) for a characterization of such a ratio-ranking in a slightly different context.
} 
which makes use of the preferences of reasonable persons. The ranking compares two sets $A$ and $B$ on the basis of the cardinality of their respective 'maximal sets' (i.e., the sets consisting of elements that are considered to be best elements within that set by at least one of the reasonable persons). In our framework, that ranking can also be seen as an instance of the simple eligibility ranking. To do so, we only have to stipulate that an element $x$ of an opportunity set $A$ is eligible, if, and only if, $x$ is a best element of $A$ according to at least one reference ordering.

Of the four axioms that we use, three can be seen as weakenings of corresponding axioms of Pattanaik and Xu (1990) since they here pertain only to the eligible elements of a set. For instance, Pattanaik and Xu's first axiom states that any two singleton sets are to be ranked equally. In our rendition of it, we demand that singleton sets with eligible elements are to be ranked equally:

Axiom 4.1 (Indifference Between Singletons (IBS)) For all distinct $x, y \in X$, if $x \in$ $E(\{x\})$ and $y \in E(\{y\})$, then $\{x\} \sim\{y\}$.

Our next axiom is a monotonicity requirement. It states that adding an element $x$ to a set $A$ will lead to an enlargement of the set's quality if the new element $x$ is eligible and if all alternatives that were eligible in $A$ remains so in $A \cup\{x\}$.

Axiom 4.2 (Monotonicity (M)) For all $A \in \mathcal{Z}$ and for all $x \in X-A$, if $E(A \cup\{x\})=$ $E(A) \cup\{x\}$, then $A \cup\{x\} \succ A$.

Obviously, this monotonicity requirement is much weaker than the assumption that any enlargement of a set will lead to a strict increase of its value. In fact, the next axiom-Weak Irrelevant Expansion-explicitly states that certain enlargements of a set do not enlarge the set's value.

Axiom 4.3 (Weak Irrelevant Expansion (WIE)) For all $A \in \mathcal{Z}$ and for all $x \in X-A$, if $E(A \cup\{x\})=E(A)$, then $A \cup\{x\} \sim A$.

Our final axiom is an independence axiom. It states that adding an alternative $x$ to a set $A$ and $y$ to a set $B$ will not affect the ranking of $A$ and $B$ vis-à-vis each other if all the elements of $A$ and $B$ as well as all the elements of $A \cup\{x\}$ and $B \cup\{y\}$ are eligible. $^{11}$

Axiom 4.4 (Restricted Composition (RC)) For all $A, B \in \mathcal{Z}$ such that $E(A)=A$ and $E(B)=B$ and for all $x \in X-A$ and for all $y \in X-B$ such that $E(A \cup\{x\})=A \cup\{x\}$ and $E(B \cup\{y\})=B \cup\{y\}$ :

$$
A \succeq B \text { iff } A \cup\{x\} \succeq B \cup\{y\} .
$$

Proposition 4.1 Let $E$ be normal. A reflexive and transitive relation $\succeq$ satisfies IBS, $M$, WIE, and $R C$ iff $\succeq=\succeq \#$.

\footnotetext{
11 The axiom is a weakening of the composition axiom used by Sen (1991). Strictly speaking, it is not a weaker version of the independence axiom used by Pattanaik and Xu since, to derive completeness of the resulting ranking, the added alternatives $x$ and $y$ may here be distinct whereas they should be the same in Pattanaik and $\mathrm{Xu}$.
} 
We will not give a complete proof of the proposition but instead only prove the following lemma which forms the crucial new element of the complete proof.

Lemma 4.1 Let $E$ be normal and let $\succeq$ be a reflexive and transitive relation satisfying WIE. For all $A \in \mathcal{Z}$ :

1. $E(A)=\emptyset: A \sim\{x\}$ for all $x \in A$;

2. $E(A) \neq \emptyset: A \sim E(A)$.

\section{Proof}

(1) $E(A)=\emptyset$. By $\xi$ and $\alpha, A$ has no subsets with eligible elements. Hence, for all $x \in A, E(\{x\})=\emptyset$. Take some $x \in A$ and add one by one all elements of $A-\{x\}$ to $\{x\}$. We then obtain $A \sim\{x\}$ by WIE and transitivity.

(2) $E(A) \neq \emptyset$. If $A=E(A)$ the result follows from reflexivity. Let therefore $A \neq$ $E(A)$. We first show that for any $B, E(A) \subseteq B \subset A$, there is an $x \in A-B$ such that $E(B)=E(B \cup\{x\})$.

(2a) Assume $E(A-B)=\emptyset$ and let $x$ be an arbitrary element of $A-B$. Since $A-B$ contains no eligible elements, $x$ is unconditionally ineligible. In particular, we have $x \notin E(B \cup\{x\})$. Hence, $E(B \cup\{x\}) \subseteq E(B)$. Take $y \in E(B)$. Since $x$ is unconditionally ineligible, $y P x$. Since we also have $y R z$ for all $z \in B$ by normality, $y R z$ for all $z \in B \cup\{x\}$. It follows that $y \in E(B \cup\{x\})$ and thus that $E(B) \subseteq E(B \cup\{x\})$.

(2b) Assume $E(A-B) \neq \emptyset$. Take $x \in E(A-B)$. By normality we have $x R y$ for all $y \in A-B$. If $x R y$ also holds for all $y \in B$, we would have $x \in E(A)$ by normality and non-emptiness of $E(A)$, contradicting $x \in A-B$ and $E(A) \subseteq B$. Hence, $y P x$ for some $y \in B$ and thus $x \notin E(B \cup\{x\})$, which means that $E(B \cup\{x\}) \subseteq E(B)$. If $x P z$ for some $z \in E(B)$, we have $y P z$ by transitivity, contradicting $z \in E(B)$. Hence, for all $z \in E(B)$ and all $y \in B \cup\{x\}, z R y$. Thus $z \in E(B \cup\{x\})$, that is, $E(B) \subseteq E(B \cup\{x\})$.

Hence, for any $B$ such that $E(A) \subseteq B \subset A$ there is an $x \in A-B$ for which $E(B \cup\{x\})=E(B)$. This means we can label the elements of $A-E(A)$ as $x_{1}, x_{2}, \ldots$, such that $E(E(A))=E\left(E(A) \cup\left\{x_{1}\right\}\right)=E\left(E(A) \cup\left\{x_{1}, x_{2}\right\}\right)=\ldots=E(A)$. If we subsequently add the elements $x_{1}, x_{2}, \ldots$ one by one to $E(A)$ we arrive at the desired result by WIE and transitivity of $\succeq$.

Proposition 4.1 can now be derived with the help of the original result of Pattanaik and $\mathrm{Xu}$ (1990). That is, their result entails that $E(A) \succeq E(B)$ iff $\# E(A) \geq \# E(B)$ for all $A, B(E(A) \neq \emptyset \neq E(B))$. The proposition follows from the lemma plus the fact that $A \succ B$ whenever $E(A) \neq \varnothing=E(B)$.

\section{The relative eligibility ranking}

Assume there is some element in $X$, say $x$, which represents a pure state of 'bliss'. Any view about what constitutes the good life would judge it to be the uniquely best of all possible worlds. Clearly the availability of such an alternative means one's choice set is much more valuable than a choice set in which the various options are merely 
satisfactory. Yet, as long as those latter options are also deemed to be eligible, such a conclusion is not ensured on the simple eligibility ranking. After all, it judges any two choice sets having the same number of eligible alternatives to be of equal value. For instance, if $x$ is the blissful option and $y$ one of average quality, we have $\{x\} \sim_{\#}\{y\}$.

We can avoid such a conclusion by considering a ranking which ranks two opportunity sets $A$ and $B$ not only on the basis of information about the eligibility of alternatives within $A$ and $B$, but also takes account of the eligibility of an alternative in the set $A \cup B$. To understand the rationale of this, consider an interpretation of eligibility in terms of the person's actual preferences: an element of $A$ is eligible if it is one of the individual's most preferred elements in $A$. The value of an available option $x-x$ 's eligibility-is determined completely by whether the element is 'the best the agent can do'; if the agent has the option of choosing a more preferred alternative, then $x$ is not eligible. On such a view about the eligibility of options, it is natural to say that a ranking of $A$ versus $B$ should depend on the eligibility of the elements of $A$ in the context of those of $B$, and vice versa. Suppose for instance that a set $A$ has two best elements and that $B$ has only one, but that the person strictly prefers the best element of $B$ to those of $A$. If only utility considerations matter, we would like to have a ranking that judges $B$ to confer more value than $A$.

The ranking which is analyzed next, and which we call the relative eligibility ranking, expresses this idea. It was first introduced by Puppe (1996) and characterized in Puppe and $\mathrm{Xu}$ (2010). It avoids the problem of the simple ranking by making a comparison between two sets $A$ and $B$ dependent on the eligible alternatives within the set $A \cup B$.

Definition 5.1 (Relative eligibility ranking) For all $A, B \in \mathcal{Z}, A \succeq_{+} B \Leftrightarrow \#(E(A \cup$ $B) \cap A) \geq \#(E(A \cup B) \cap B)$.

Our characterization of this ranking differs in several respects from Puppe's and Xu's. First of all, we should allow for the possibility that an opportunity set does have not any eligible elements whereas this is precluded in Puppe's and Xu's framework. Second, an important difference concerns the assumptions that are being made. We here examine the conditions under which a reflexive and transitive relation over $\mathcal{Z}$ coincides with the relative eligibility ranking. Puppe's and Xu's main result, on the other hand, states the conditions under which any binary relation coincides with it. Clearly, the restriction to the class of transitive and reflexive binary relations limits the generality of our results but it has the advantage of allowing us to use weaker conditions in the derivation of our result. In fact, it enables us to clearly see the difference between the characterization of the relative ranking and that of the simple ranking.

Indeed, we obtain our next result by dropping the axiom IBS and by strengthening two of the other axioms used to characterize $\succeq_{\#}$. In particular, we strengthen WIE and $\mathrm{M}$ :

Axiom 5.1 (Irrelevant Expansion (IE)) For all $A \in \mathcal{Z}$ and for all $x \in X-A$, if $x \notin E(A \cup\{x\})$, then $A \cup\{x\} \sim A$.

Axiom 5.2 (Strong Monotonicity $(S M)$ ) For all $A \in \mathcal{Z}$ and for all $x \in X-A$, if $x \in E(A \cup\{x\})$, then $A \cup\{x\} \succ A$. 
Before we proceed we note that these strengthenings are difficult to combine with IBS. According to IE and SM, the effect of an expansion of a set is only dependent on the eligibility of the added element. Because of path-dependencies, the conditions will in combination with IBS lead to a violation of transitivity if there is context-dependent eligibility:

Proposition 5.1 Let $E$ be normal. A reflexive and transitive relation $\succeq$ satisfies IBS, $I E$, and SM only if each element in $X$ is either unconditionally eligible or unconditionally ineligible.

Proof It is easily verified that if some $x \in X$ is not unconditionally eligible or ineligible, we have some $C \in \mathcal{Z}$ such that $x \in E(C)$ and $x \notin E(X)$. By normality of $E$ there is some $y \in E(X)-C$ such that $E(\{x, y\})=\{y\}$. By $\alpha$ and $x \in E(C)$ we have $E(\{x\})=\{x\}$. Similarly, $E(\{x, y\})=\{y\}$ entails $E(\{y\})=\{y\}$. Hence, $\{x\} \sim\{y\}$ by IBS. However, $E(\{x, y\})=\{y\}$ implies $\{x, y\} \succ\{x\}$ by SM, and $\{x, y\} \sim\{y\}$ by IE, violating transitivity.

We next present two lemmas, the first of which is a straightforward application of RC:

Lemma 5.1 If a reflexive relation $\succeq$ satisfies $R C$, then $x \in E(\{x\}), y \in E(\{y\})$, and $\{x, y\}=E(\{x, y\})$ implies $\{x\} \sim\{y\}$.

Lemma 5.2 Let $E$ be normal and $R$ its base relation. If a transitive relation $\succeq$ satisfies $I E, S M$, and $R C$, then $R$ is an ordering.

Proof Completeness and reflexivity of $R$ follows directly from $\alpha$ and the definition of $R$. To prove transitivity, assume for some $x, y, z \in X$, we have $x R y$ and $y R z$. We prove by contradiction: let $z P x$, that is, $E(\{x, z\})=\{z\}$. We need to consider the following cases:

(a) $E(\{x, y\})=\{x, y\}$. Property $\alpha$ implies non-empty $E(\{y\})$ and thus by $\xi$ nonempty $E(\{y, z\})$. Given $y R z$, either (a1) $E(\{y, z\})=\{y, z\}$ or (a2) $E(\{y, z\})=$ $\{y\}$. Assume (a1). By $\gamma$ and $\alpha, E(\{x, y, z\})=\{y, z\}$. IE implies $\{x, y, z\} \sim\{y, z\}$ and $\operatorname{SM}\{x, y, z\} \succ\{x, y\}$. By Lemma $5.1\{x\} \sim\{y\}$ and by $\operatorname{RC}\{x, y\} \sim\{y, z\}$, violating transitivity of $\succeq$. Consider next (a2). By $\alpha$ and $x \notin E(\{x, z\}), x \notin$ $E(\{x, y, z\})$. Similarly, $\alpha$ and $z \notin E(\{y, z\})$, entails $z \notin E(\{x, y, z\})$. Since $E(\{x, y, z\}) \neq \varnothing$ by $\xi$, we have $E(\{x, y, z\})=\{y\}$. IE entails $\{x, y, z\} \sim$ $\{y, z\} \sim\{y\}$, and $\{x, y, z\} \sim\{x, y\}$. By $\operatorname{SM}\{x, y\} \succ\{y\}$, violating transitivity of $\succeq$.

(b) $E(\{x, y\})=\{x\}$. By $\alpha, x, y \notin E(\{x, y, z\})$. By $\xi$ we have non-emptiness of $E(\{x, y, z\})$, and thus $E(\{x, y, z\})=\{z\}$. It follows from $\alpha$ that $z \in E(\{y, z\})$. Hence, $E(\{y, z\})=\{y, z\}$ because of $y R z$. With IE we derive $\{x, y, z\} \sim$ $\{x, z\} \sim\{z\}$ and $\{x, y, z\} \sim\{y, z\}$. By $\operatorname{SM}\{y, z\} \succ\{z\}$ violating transitivity of $\succeq$.

(c) $E(\{x, y\})=\emptyset$. We then have $E(\{x, y, z\})=\{z\}$ by $\xi$ and $\alpha$. By $\alpha, E(\{y, z\})=$ $\{z\}$, contradicting $y R z$.

Proposition 5.2 Let $E$ be normal. A reflexive and transitive relation $\succeq$ satisfies IE, $S M$, and $R C$ iff $\succeq=\succeq+$. 
Proof

(a) $\Leftarrow$ : The proofs of SM and of the necessity part of RC are straightforward and therefore omitted. To prove that $\succeq_{+}$satisfies IE and the sufficiency part of RC, it is useful to note that for all $x, y \in X,\{x\} \succeq_{+}\{y\}$ iff $x R y$, where $R$ is the base relation. Transitivity of $\succeq_{+}$thus entails transitivity of $R$, which in turn means that $\beta$ is satisfied (Proposition 3.2).

IE: Take $A \in \mathcal{Z}$ and $x \in X-A$ for which $x \notin E(A \cup\{x\})$. If $E(A \cup\{x\})=\emptyset$, we see directly that $A \cup\{x\} \sim_{+} A$. Let $E(A \cup\{x\}) \neq \emptyset$. Since $x \notin E(A \cup\{x\})$, we have $E(A \cup\{x\}) \cap A \neq \emptyset$. By $\beta$, we then have $E(A \cup\{x\}) \cap A=E(A)$ and, because $x \notin E(A \cup\{x\}), E(A \cup\{x\}) \cap(A \cup\{x\})=E(A)$. Hence, $A \cup\{x\} \sim_{+} A$.

$R C$ : Let $A, B$ be opportunity sets such that for some $x \in X-A$ and $y \in X-B$ we have $E(A \cup\{x\})=A \cup\{x\}$ and $E(B \cup\{y\})=B \cup\{y\}$. Assume $A \succeq_{+} B$. It has to be shown that $A \cup\{x\} \succeq_{+} B \cup\{y\}$. If $E(A \cup B \cup\{x, y\}) \cap(B \cup\{y\})=\varnothing$, the result follows immediately. Therefore, let $E(A \cup B \cup\{x, y\}) \cap(B \cup\{y\}) \neq \emptyset$. Because $E(B \cup\{y\})=B \cup\{y\}$, we have, by $\beta, E(A \cup B \cup\{x, y\}) \cap(B \cup\{y\})=B \cup\{y\}$. By $\alpha$ and $\beta$, we have $E(A \cup B) \cap B=E(B)=B$. Since $A \succeq_{+} B$, $E(A \cup B) \cap A \neq \emptyset$. In particular, we then have by $\beta, E(A \cup B) \cap A=E(A)=A$. Non-emptiness of $E(A \cup B \cup\{x, y\}) \cap(A \cup B)$ implies by $\alpha$ and $\beta$ non-emptiness of $E(A \cup B \cup\{x, y\}) \cap A$ and thus also of $E(A \cup B \cup\{x, y\}) \cap(A \cup\{x\})$. By $\alpha$ and $\beta, E(A \cup B \cup\{x, y\}) \cap(A \cup\{x\})=E(A \cup\{x\})=A \cup\{x\} . A \succeq_{+} B$ implies $\#(E(A \cup B) \cap A)=\# E(A)=\# A \geq \#(E(A \cup B) \cap B)=\# E(B)=\# B$. But

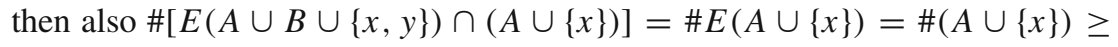
$\#[E(A \cup B \cup\{x, y\}) \cap(B \cup\{y\})]=\# E(B \cup\{y\})=\#(B \cup\{y\})$.

(b) $\Rightarrow$ : Let $A$ and $B$ be two sets with $\# E(A \cup B) \cap A \geq \# E(A \cup B) \cap B$.

Case $1 \# E(A \cup B) \cap A=\# E(A \cup B) \cap B=0$.

By $\alpha, E(A \cup B)=\emptyset$. We thus have to show that $A \sim B$. By $\xi$ and Lemma 4.1 we can take some $x \in A$ and $y \in B$ such that $E(\{x\})=\emptyset=E(\{y\})$ and $A \sim\{x\}$ and $B \sim\{y\}$. From $E(\{x\})=\emptyset=E(\{y\})$ we have, by $\alpha, E(\{x, y\})=\emptyset$. Hence, again by Lemma 4.1, $\{x\} \sim\{x, y\} \sim\{y\}$. $A \sim B$ now follows from transitivity.

Case $2 E(A) \neq \emptyset=E(B)$.

By $\alpha, E(A \cup B) \cap B=\emptyset$. Since therefore $E(A \cup B) \cap A \neq \emptyset$, it has to be shown that $A \succ B$.

As $E(B)=\varnothing$, we have by Lemma 4.1 and $\xi$ for all $x \in B E(\{x\})=\varnothing$ and $B \sim\{x\}$. Take $y \in E(A \cup B) \cap A$. By $\alpha, y \in E(A)$ and $y \in E(\{x, y\})$. By $\alpha$ also $x \notin E(\{x, y\})$ and $y \in E(\{y\})$, and by SM thus $\{x, y\} \succ\{x\}$ and $\{x, y\} \sim\{y\}$. Hence, by transitivity $\{y\} \succ B$. Now add one by one the other elements of $E(A)$ to $\{y\}$. By $\alpha$ each such added element is eligible in the new set, and thus by repeated application of SM and transitivity $E(A) \succ\{y\}$. Lemma 4.1 shows that $A \sim E(A)$. Applying transitivity one more time we get $A \succ B$. 
Case $3 E(A \cup B) \cap B \neq \emptyset$. By $\alpha$ we then have $E(B) \neq \emptyset$. Since \#E( $A \cup B) \cap A \geq$ $\# E(A \cup B) \cap B$, we then also have $E(A \cup B) \cap A \neq \emptyset \neq E(A)$. By Lemma 5.2 the base relation $R$ is an ordering and $\beta$ is therefore satisfied.

By $\beta, E(A)=E(A \cup B) \cap A$ and $E(B)=E(A \cup B) \cap B$. Given Lemma 4.1, it therefore suffices to show that $E(A) \sim E(B)$ if $\# E(A)=\# E(B)$ and $E(A) \succ E(B)$ if $\# E(A)>\# E(B)$. Take $x \in E(A)$ and $y \in E(B)$. Since $x, y \in$ $E(A \cup B)$ we have, by $\alpha, E(\{x, y\})=\{x, y\}, E(\{x\})=\{x\}$ and $E(\{y\})=\{y\}$. Hence, $\{x\} \sim\{y\}$ by Lemma 5.1. Adding \#B-1 elements of $E(A)$ one by one to $\{x\}$ and the remaining elements of $B-\{y\}$ one by one to $y$, yields by RC $C \sim B$, where $C$ is the subset resulting from adding the $\# B-1$ elements of $E(A)$ to $\{x\}$. If $C=E(A)$, we have the first desired result. If $C \neq E(A)$, adding the remaining elements of $E(A)$ to $C$ yields by SM and transitivity, $E(A) \succ C$. Hence, by transitivity also $E(A) \succ E(B)$.

The assumption of transitivity of $\succeq$ had a significant impact on the results. Puppe and $\mathrm{Xu}$ show (2010, Proposition 4.5) that if an eligibility function $E$ assigns a nonempty subset to any opportunity set, then the relation $\succeq_{+}$is transitive if, and only if, $E$ satisfies $\alpha$ and $\beta$. Clearly, condition $\beta$ is then rather demanding since it entails that an eligibility function can always be rationalized by a unique preference ordering. Indeed, if we drop the assumption of transitivity we find rankings which satisfy the axioms but which do not coincide with $\succeq_{\#}$ or $\succeq_{+}$:

Example $X=\{x, y\}, E(\{x, y\})=\{x\}, E(\{x\})=\{x\}$, and $E(\{y\})=\emptyset$. Assume $\{x, y\} \succ\{y\},\{x, y\} \sim\{x\}$, and $\{x\} \sim\{y\}$. E satisfies $\alpha, \beta, \gamma$, and $\xi$ and $\succeq$ satisfies all of the axioms used so far. However, $\succeq_{\# \neq} \neq \neq \succeq_{+}$.

We conclude this section by noting that the simple and relative eligibility ranking will coincide in very special cases only. Indeed, it follows from Proposition 5.1 that they do so only if each element of $X$ is unconditionally eligible or unconditionally ineligible.

\section{Conclusion}

We have explored various ways of capturing the specific value of opportunity sets, that is, the value that we assign to the freedom to be able to pick an element from a set insofar as that value can be reduced to the value of the elements of the set. The framework makes use of eligibility functions, which were introduced in the formal literature by Puppe (1996, 1998).

The simple and relative eligibility ranking have an important feature in common. Both are cardinal measures; they are based on a count of the number of eligible elements in a set. An element's eligibility was taken as indicative of the fact that a person has reason to value that alternative. As is well known, a cardinal approach may yield counterintuitive conclusions since it does not take account of the similarities or dissimilarities within a set (Pattanaik and Xu 1990; Van Hees 2004). Suppose $x$ is an option which is eligible, i.e., an alternative a person has reason to value. Say $x$ is a can of orange juice and enlarge the person's opportunity set with another can of juice, $y$, which only differs from $x$ in its bar code. Clearly, the person will value $y$ for 
exactly the same reasons as he values $x$ - the fact that the juice comes in a can with a different bar code does not provide a new reason to value it. The specific value of the opportunity set has not increased even though the set has been enlarged with an eligible element. However, this means that both the simple and the relative eligibility ranking may overstate a set's specific value. After all, the addition does lead to a strict increase of the opportunity set's value in both rankings.

We do not want to discuss here possible solutions to the problem of finding a measure of freedom that is sensitive to the degree of diversity within a set. ${ }^{12}$ However, we do think that the need to incorporate diversity considerations naturally suggests a multi-dimensional extension of the framework. To see why, note that we may deem diversity to be important because, first, we may not yet know the reason why we value the alternatives; we may call this the epistemic consideration. That is, even though we should now assign a value to an opportunity set, the eligibility of its elements can only be assessed at some later point in time. An illustration of this perspective is the preference-for-flexibility approach discussed in the introduction. If we say that the eligibility of an alternative is determined by whether it realizes our future preferences, then in the absence of information about the exact nature of those future preferences we can at best assume the existence of multiple eligibility functions, representing judgements we can potentially make. Second, we may value a set's diversity because it points out that eligibility can have a multi-dimensional nature. Consider, for instance, Nussbaum's capability theory according to which a person is able to live a life of dignity if he is able to choose particular functionings (Nussbaum 2006). If the alternatives are taken to be vectors of functionings, we can formulate for each relevant functioning an eligibility function which describes which elements of a set realize that particular functioning. In that case there are as many eligibility functions as there are different functionings, or more generally, as there are different reasons for valuing an outcome. As in the epistemic case, we then assume the existence of a family of normal eligibility functions and can formulate extensions of our rankings to this multi-dimensional setting. In the epistemic case, the extensions of the eligibility rankings would be based on the maximal number of eligibility considerations (different future preferences) that the set can accommodate for. In the interpretation in which eligibility itself is multidimensional, they will be based on the maximal number of eligibility considerations (e.g., functionings one has reason to value) that specific elements of the set satisfy.

A second important issue is whether the eligibility framework can be used to capture freedom's non-specific value. It is particularly interesting to explore the question whether this part of freedom's value requires the abandonment of the assumption of normality of eligibility functions. ${ }^{13}$ Sen's celebrated examples pointing out the possible non-normality of choice functions may be helpful here. Take, for instance, an alternative which in itself is ineligible (say starvation) but which becomes eligible if a person has the option of choosing the opposite state of affairs (i.e., it becomes fasting rather than starvation). The example could be used to point out that our freedom has

\footnotetext{
12 For important contributions, see Pattanaik and Xu (2000), Nehring and Puppe (2002), Bossert et al. (2003), and Bervoets and Gravel (2007).

13 For a discussion of dropping the assumption of normality of eligibility functions in the context of the ranking of capability sets, see Binder (2009).
} 
a value which originates from the act of choosing itself, rather than from the contents of our choices. Similarly, and returning to the argument discussed in the introduction, we may value having the option to choose a non-valuable alternative because the alternative's availability creates responsibility. If such a responsibility-generating effect can only occur if there are other choice options, the element $x$ would be eligible in a set $A$ which contains other elements as well but not in the singleton set $\{x\}$. Clearly, we then have a violation of normality. Furthermore, as is well known from the literature on choice functions, since non-normal eligibility functions cannot be rationalized by orderings, we have a further argument for using the more general framework of eligibility functions rather than a preference-based approach.

A further interesting question to explore is whether the 'eligibility approach' allows us to derive judgements about the measurement of the value of our freedom in a social context. Except for Gravel et al. (1998) and, more recently, Bervoets (2007), no formal work has been done on this issue. An obvious possibility is to say that we then want to rank games rather than opportunity sets, and to associate with each game the set of its equilibria as the set of its eligible outcomes.

Acknowledgments I am very grateful to two anonymous referees for their detailed comments and suggestions. I also thank Constanze Binder, Clemens Puppe, and Yongsheng Xu for their comments on an earlier version of this article.

Open Access This article is distributed under the terms of the Creative Commons Attribution Noncommercial License which permits any noncommercial use, distribution, and reproduction in any medium, provided the original author(s) and source are credited.

\section{References}

Arrow KJ (1995) A note on freedom and flexibility. In: Basu K, Pattanaik PK, Suzumura K (eds) Choice, welfare, and development. A Festschrift in honor of Amartya K Sen. Clarendon, Oxford, pp 7-16

Barberà S, Bossert W, Pattanaik PK (2004) Ranking sets of objects. In: Barberà S, Hammond PJ, Seidl Chr (eds) Handbook of utility theory, vol 2: extensions. Kluwer, Dordrecht, pp 893-978

Benn SI, Weinstein WL (1971) Being free to act, and being a free man. Mind 80:194-211

Bervoets S (2007) Freedom of choice in a social context: comparing game forms. Soc Choice Welf 29:295315

Bervoets S, Gravel N (2007) Appraising diversity with an ordinal notion of similarity: an axiomatic approach. Math Soc Sci 53:259-273

Binder C (2009) Context dependency of valuable functionings: how culture affects the capability framework. In: Chiappero-Martinetti E (ed) Debating global society: reach and limits of the capability approach. Fondazione Giangiacomo Feltrinelli, Milan , pp 205-232

Bossert W, Pattanaik PK, Xu Y (2003) Similarity of options and the measurement of diversity. J Theor Politics 15:405-421

Carter I (1996) The concept of freedom in the work of Amartya Sen: an alternative analysis consistent with freedom's independent value. Notizie di Politeia 12:7-22

Carter I (1999) A measure of freedom. Oxford University Press, Oxford

Day JP (1977) Threats, offers, law, opinion and liberty. Am Philos Q 14:257-272

Dowding K, van Hees M (2009) Freedom of choice. In: Anand P, Pattanaik PK, Puppe C (eds) The Oxford handbook of rational and social choice. Oxford U.P., Oxford , pp 374-392

Foster J (1992) Notes on effective freedom. Mimeo

Frankfurt H (1969) Alternate possibilities and moral responsibility. (Reprinted from The importance of what we care about. Philosophical essays, 1988.) Cambridge University Press, Cambridge, pp 1-10 
Gravel N, Laslier J-F, Trannoy A (1998) Individual freedom of choice in a social setting. In: Laslier JF, Fleurbaey M, Gravel N, Trannoy A (eds) Freedom in economics new perspectives in normative analysis. Routledge, London , pp 76-92

Griffin J (2008) On human rights. Oxford University Press, Oxford

Jones P, Sugden R (1982) Evaluating choice. Int Rev Law Econ 2:47-65

Kreps DM (1979) A representation theorem for preference for flexibility. Econometrica 47:565-577

Nehring K, Puppe C (2002) A theory of diversity. Econometrica 70:1155-1198

Nussbaum M (2006) Frontiers of justice. Disability, nationality, species membership. Harvard University Press, Harvard

Pattanaik PK, Xu Y (1990) On ranking opportunity sets in terms of freedom of choice. Récherches Economiques de Louvain 56:383-390

Pattanaik PK, Xu Y (1998) On preference and freedom. Theory Decis 44:173-198

Pattanaik PK, Xu Y (2000) On diversity and freedom of choice. Math Soc Sci 40:123-130

Puppe C (1996) An axiomatic approach to "preference for freedom of choice". J Econ Theory 68:174-199

Puppe C (1998) Individual freedom and social choice. In: Laslier J-F et al (ed) Freedom in economics new perspectives in normative analysis. Routledge, London , pp 49-68

Puppe C, Xu Y (2010) Essential alternatives and freedom rankings. Soc Choice Welf, this issue

Sen AK (1971) Choice functions and revealed preference. Rev Econ Stud 38:307-317

Sen AK (1985) Commodities and capabilities. North Holland, Amsterdam

Sen AK (1987) On ethics and economics. Blackwell, Oxford

Sen AK (1991) Welfare, preference and freedom. J Econom 50:15-29

Sen AK (1996) Freedom, capabilities and public action: a response. Notizie di Politeia 12:107-125

Sen AK (2004) Rationality and freedom. Belknap Press, Harvard

Steiner H (1994) An essay on rights. Blackwell, Oxford

Sugden R (1998) The metric of opportunity. Econ Philos 14:307-337

Van Hees M (1998) On the analysis of negative freedom. Theory Decis 45:175-197

Van Hees M (2000) Legal reductionism and freedom. Kluwer, Dordrecht

Van Hees M (2004) Freedom of choice and diversity of options: some difficulties. Soc Choice Welf 22:253266 